\title{
Idiosyncratic response of yeasts, recovered from oropharyngeal lesions of HIV patients, to prophylactic antifungal agents, in South Western Uganda
}

Ezera -Agwu ( $\sim$ editorialoffice@spparenet.us )

Research article

Keywords:

Posted Date: September 11th, 2019

DOI: https://doi.org/10.21203/rs.2.14270/v1

License: (9) (1) This work is licensed under a Creative Commons Attribution 4.0 International License. Read Full License 


\section{Abstract}

Background: Although cutting edge technologies have advanced overall knowledge of microbial infections, what microorganisms will do when challenged with surface active agents is incompletely understood and understanding the characteristic uniqueness of an organism when challenged with antimicrobial agents derails antibiotic stewardship programs in resource limited settings. T his study was therefore designed to determine the idiosyncratic in-vitro response of yeast strains, challenged with selected routine antifungal agents used for routine prophylactic management of HIV infected Methods: A total of 304 (235 females and 69 males) patients with different conditions were recruited. The analysis performed included: cultivation on Sabouraud Dextrose Agar, Corn Meal Agar, Potato Dextrose Agar, germ-tube test, chlamydospore production, test for ability to grow at $45 \circ \mathrm{C}$. For further morphologic identification, yeasts were grown on CHROMagar Candida medium at $37+20 \mathrm{C}$ for 24-48 hours and subcultured on Pal's agar. The minimum inhibitory concentration (MIC) of the isolates were determined by the E-test stable agar gradient method (AB BIODISK, Solna, Sweden) Results: The 304 (235 females and 69 males) recruited patients with different oral lesions consented to this study and 304 sample surveyed was guided by the upper limit required to give $95 \%$ level of confidence at an expected prevalence of about 74\% (Agwu et al, 2008) using : Sample size (N) = Z 2 P (100-P)/D2 giving 296 samples, (Epi-info version 3.2 data-base; 1995), where $Z$ is a constant given as (1.96), $P$ is expected prevalence $(74 \%)$, and $D$ is acceptable error $(5 \%)$. Informed consent was sought and obtained from the following: Uganda National Council of Science and Technology, Kampala International University Research and Ethics Committee, HIV patients of The AIDS Support Organizations,. Conclusion : Candida albicans remain significantly susceptible to tested antifungal agents. Emerging dose dependent susceptibility in non albicans oropharyngeal yeast strains is interesting in this era of increasing treatment failures due to drug resistance. Pattern of resistance recorded may suggest significant contribution of yeast in the recurrent or delayed oropharyngeal lesions of HIV population studied. Sustained surveillance with MIC which impacts treatment outcome is recommended

\section{Background}

Oropharyngeal Candidiasis (OC) is one of the Acquired immunodeficiency syndrome (AIDS) defining opportunistic infections caused by Human Immunodeficiency viruses (HIV). Recurrent oropharyngeal Candidiasis common among HIV sero-positive patients may be associated to: drug-resistance-related treatment failure (1); non-compliance to drug prescription guidelines (2); use of sub-lethal drug concentration (3-4); re-infection of microbial disease aetiology in disease Endemic regions (5) or unruffled progression of HIV disease to AIDS (1-2). To succeed in oropharyngeal lesion management in the immune-suppressed HIV disease, intervention must be based on what is known about the population structure of the oral microecology and yeast resistance to treatment regimen (6-9). This should then give rise to bases for new drug development plans, in answer to identified challenges and unmet therapeutic needs so that sustainable intervention for today and tomorrow outbreaks can be contained (10) 
To reduce rates of treatment failure, systematic treatment should take place during the fungal growth phase when constant breakdown and re-synthesis of cell walls constituent giving polyenes more access to the sensitive targets of host cells (11). Polyenes and Azoles remain the antifungal agents most commonly used to treat Candida infections in the immunocompromised patients living in Uganda. The polyenes like amphotericin $B$ and nystatin are fungicidal agents that bind preferentially to the ergosterol found in fungal membranes (12-13). This disrupts the integrity of the cellular membrane, causing cell death. Resistance to polyenes may develop by modifying the ergosterol molecule or its membrane content and mechanisms of yeasts cells resistance to polyenes include replacement of the polyenebinding ergosterol, cholesterol, or stigmasterol with polyene-less binding 3-hydroxy or 3-oxo sterols (1, 34).. Fluconazole and ketoconazole are fungistatic agents that inhibit cytochrome P450 14a-demethylase, a component of the sterol biosynthesis pathway $(11,14-16)$. New azole and triazole derivatives [voriconazole, ER-30346, and D0870] and SCH-56592, which is a hydroxylated analogue of Itraconazole may be available but they are not reachable to the population that need them in resource limited counties like Uganda.

Accepting to live with HIV (17) may be understandable because HIV infection have no clear routine cure yet, but no one would accept to live with recurrent oropharyngeal lesions associated with yeast strains when presumed cure are available (18). Management of HIV infected patients with recurrent oropharyngeal lesions remain a challenge because of the inherent dilemma associated with persistence of infection. Thus, It is not easy to determine the cause attribution factors to either HIV disease progression (19) or treatment failure associated with resistance of microbial oral pathogens found in the microecology of the oral cavity $(1,8-9,20)$. There is no anti-fungal medication widely available for the majority of HIV-infected patients with oral lesions in Uganda. Majority of HIV patients in Uganda are on prophylactic drugs depending on the disease staging status. They are either routinely given antibacterial, antifungal or antiviral either separately of together.

There is no organized surveillance to update healthcare managers with performance of antimicrobial agents used in prophylactic management of HIV associated diseases. While in vivo assessment of drug performance when administered to sick people to treat associated infections remain relevant, such experiment depends on enough representative in-vitro data. This study was therefore designed to determine the idiosyncratic in-vitro response of yeast strains, challenged with selected routine antifungal agents used for routine prophylactic management of HIV infected TASO patients in Uganda, with the ultimate goal of ascertaining the performance of the prophylactic medications and impacting the outcome of future intervention.

\section{Methods}

Three TASO centres in Masaka, Rukunguri, and Mbarara districts of were used for this investigation. Patient's sampled were TASO HIV patients with clinically diagnosed oral lesions and receiving cotrimoxazole, Nystatine and clotrimazole prophylaxis. Approvals were obtained from Uganda National Council of Sciences and technology, The AIDS Support Organizations (TASO), TASO clients (patients) 
through their informed consent. A total of 304 (235 females and 69 males) patients with different oral lesions consented to participate in this study and were therefore recruited. This 304 sample size surveyed was guided by the upper limit required to give $95 \%$ level of confidence at an expected prevalence of about $74 \%$ (6) using the precise prevalence formula: Sample size $(N)=Z^{2} P(100-P) / D 2$ giving 296 samples, (Epi-info version 3.2 data-base; 1995), where $Z$ is a constant given as (1.96), $P$ is expected prevalence (74\%), and D is acceptable error (5\%). Eight (8) more samples was added arbitrarily just in case of drop out and unseen error making a total of 304 samples. The mouth examination of the TASO clients was done by previously trained and calibrated oral clinicians according to WHO standards (21).

All analyses for yeast identification were done at the Microbiology Department, Kampala International University Western Campus. The analysis performed included: cultivation on Sabouraud Dextrose Agar, Corn Meal Agar, Potato Dextrose Agar, germ-tube test, chlamydospore production, test for ability to grow at $45^{\circ} \mathrm{C}$. For further morphologic identification of yeasts, they were first grown on CHROMagar Candida medium at $37 \pm 2^{0} \mathrm{C}$ for $24-48$ hours and sub-cultured on Pal's agar. (22). Suspect colonies of yeast strains were further identified and differentiated by biochemical test using sugar assimilation test pattern (ID32 C) for Candida species provided by BioMereux ${ }^{(\mathrm{R})}$ Paris, France.

\section{Determination of MIC of fungal Isolates by E-test}

The minimum inhibitory concentration (MIC) of the isolates were determined by the E-test stable agar gradient method (AB BIODISK, Solna, Sweden) which has been standardized and comparable with Clinical Laboratory Standard Institute (CLSI) and EUropean Committee for Antimicrobial Susceptibility Testing (EUCAST) (23-24). This involved placing a plastic strip containing a gradient of an antifungal agent on the surface of an inoculated $\mathrm{RPMI}^{(\mathrm{R})}$ agar plate. The drug diffuses into the $\mathrm{RPMI}^{(\mathrm{R})}$ agar and establishes a stable concentration gradient. Inhibition of fungal growth produces an ellipse, and the MIC is read where the ellipse intersects the test strip.

The Randomized Block Design (RBD) and Completely Randomized Design (CRD) ( $a=0.1$ or $99 \%$ level of confidence) summarized as [Total sum of squares $\left(\mathrm{SS}_{\mathrm{T}}\right)=$ Sum of squares due to different effects $\left(\mathrm{SS}_{\mathrm{D}}\right)$ + Error sum of effects $\left(\mathrm{SS}_{\mathrm{E}}\right)$ ] (25), for one observation per treatment were used to test for the relationship between each yeast strains isolated and the district of isolation and between each yeast strains isolated and antifungal agents in different districts studied. The relation between each yeast response to antifungal used were compared to responses from other yeasts within same district. Fisher's least significant difference (FLSD; $a=0.05$ ) and Duncan's Multiple Range test (DMRT; $a=0.01$ ) were used to test for levels of significance of any relationship identified after computation with CRD and RBD.

\section{Results}

From (Table 1) below, one hundred and forty four (144) yeast isolates, obtained from the oropharyngeal lesions of consenting 304 HIV patients on routine co-trimoxazole, Nystatine and clotrimazole prophylaxis comprised of: 100 Candida albicans (34 from Mbarara, 33 were from Masaka and 33 from Rukungiri); 10 
C glabrata (4 from Mbarara, 3 were from Masaka and 3 from Rukungiri), 3 C sake ( 2 from Mbarara, 1 were from Masaka and 0 from Rukungiri), $6 \mathrm{C}$ parapsilosis (2 from Mbarara, 2 were from Masaka and 2 from Rukungiri), $10 \mathrm{C}$ norevegiensis (4 from Mbarara, 3 were from Masaka and 3 from Rukungiri), $5 \mathrm{C}$ tropicalis ( 3 from Mbarara, 1 was from Masaka and 1 from Rukungiri), 3 Saccharomyces kluverii (1 from Mbarara, 0 were from Masaka and 2 from Rukungiri), 5 Saccharomyces cerevesie ( 3 from Mbarara, 2 were from Masaka and 0 from Rukungiri), and 2 Zygosaccharomyces species ( 1 from Mbarara, 0 were from Masaka and 1 from Rukungiri) (Table 1).

MIC Result interpretation, based on CLSI formerly (NCCLS) guideline for antifungals were as follows: Fluconazole: $\mathrm{MIC}<8 \mu \mathrm{g} / \mathrm{ml}=$ sensitive; Fluconazole: $\mathrm{MIC}>8 \mu \mathrm{g} / \mathrm{ml}$ and $\leq 32 \mu \mathrm{g} / \mathrm{ml}=$ sensitive dose dependent (SDD) and $\mathrm{MIC}>32 \mu \mathrm{g} / \mathrm{ml}=$ resistant. For Itraconazole: $\mathrm{MIC} \leq 0.125 \mu \mathrm{g} / \mathrm{ml}=$ sensitive; $\mathrm{MIC}>$ $0.125 \mu \mathrm{g} / \mathrm{ml}$ and $\leq 0.5 \mu \mathrm{g} / \mathrm{ml}=$ sensitive dose dependent and $\mathrm{MIC}>0.5 \mu \mathrm{g} / \mathrm{ml}=$ Resistant. For Amphotericin B: Candida species isolates with Amphotericin B with $\mathrm{MIC}>0.5 \mu \mathrm{g} / \mathrm{ml}$ should be considered resistant because specific breakpoints have not been proposed and intra-laboratory variations mandates the use of reference isolates with known resistance.

Figures 1-6 shows representative pictures of yeast response pattern to tested antifungals and so formed the basis for which different yeast strains were declared either sensitive, sensitive dose dependent or resistant to fluconazole, Itraconazole and amphotericin B respectively. The average MIC results across the Districts surveyed, show that all Candida albicans strains were sensitive to the azoles except in Masaka District where $C$ albicans showed sensitive-dose dependent MIC of $(0.14 \mu \mathrm{g} / \mathrm{ml})$ to Itraconazole. In selection, Amphotericin B in Masaka showed sensitive MIC of $0.125 \mu \mathrm{g} / \mathrm{ml}$ while C. glabrata was: resistant to Itraconazole (MIC $>32.0 \mu \mathrm{g} / \mathrm{ml})$; and its sensitivity to Fluconazole $(\mathrm{MIC}=12.0 \mu \mathrm{g} / \mathrm{ml})$ was dose dependent in all districts surveyed. (Table 1)

Candida sake showed a uniform dose dependent sensitivity to Itraconazole (MIC $=0.5 \mu \mathrm{g} / \mathrm{ml}$ ), fluconazole ( $\mathrm{MIC}=0.9 \mu \mathrm{g} / \mathrm{ml})$ and Amphotericin B $(0.25 \mu \mathrm{g} / \mathrm{ml})$. C. parapsilosis showed uniform sensitivities to Itraconazole and Fluconazole (MICs $=0.01 \mu \mathrm{g} / \mathrm{ml}$ and $0.38 \mu \mathrm{g} / \mathrm{ml}$ ). C. norevegensis showed uniform resistance to both Amphotericin B $(\mathrm{MIC}=0.75 \mu \mathrm{g} / \mathrm{ml})$ and Itraconazole (mean $\mathrm{MIC}=2.0$ $\mu \mathrm{g} / \mathrm{ml})$. However, $C$. norvegensis sensitivity to fluconazole $(\mathrm{MIC}=8.0)$ was dose dependent. $C$. tropicalis and Saccharomyces kluyverii was sensitive to all the antifungals used in all 4 Districts with mean MICs ranging from $0.05 \mu \mathrm{g} / \mathrm{ml}$ to $1.0 \mu \mathrm{g} / \mathrm{ml}$. Saccharomyces cerevesiae was sensitive to Fluconazole (MIC = $2.0 \mu \mathrm{g} / \mathrm{ml}$ ) and resistant to Amphotericin B (mean MIC $=0.9 \mu \mathrm{g} / \mathrm{ml}$ ) in all the Districts. Finally, Zygosaccharomyces species was uniformly resistant to Itraconazole ( $\mathrm{MIC}=>32 \mu \mathrm{g} / \mathrm{ml})$ and sensitive to Fluconazole $(\mathrm{MIC}=0.75 \mu \mathrm{g} / \mathrm{ml})$ in all Districts surveyed $($ Table 1$)$

When MIC results within each District and among the 9 representative yeast strains tested were compared, $C$. parapsilosis in Mbarara District, showed more sensitivity to Itraconazole, (MIC $=0.01$ $\mu \mathrm{g} / \mathrm{ml}$ ). This sensitivity was followed by: $C$. albicans and $C$. tropicalis (MIC $=0.05 \mu \mathrm{g} / \mathrm{ml}$ each). $C$. sake $(\mathrm{MIC}=0.5 \mu \mathrm{g} / \mathrm{ml})$ showed dose dependent sensitivity.C. glabrata, $C$. norvegensis and 
Zygosaccharomyces $s p$ were all resistant to Itraconazole with MIC values of $>32.0 \mu \mathrm{g} / \mathrm{ml}, 2.0 \mu \mathrm{g} / \mathrm{ml}$ and $>32.0 \mu \mathrm{g} / \mathrm{ml}$ respectively.

In Mbarara District, all the representative 9 yeast strains were sensitive to Fluconazole with Candida sake being the most sensitive $(0.09 \mu \mathrm{g} / \mathrm{ml})$ followed by: C. parapsilosis $(0.38 \mu \mathrm{g} / \mathrm{ml}) ;$ C . albicans $(0.5 \mu \mathrm{g} / \mathrm{ml})$; Zygosaccharomyces species $(0.75 \mu \mathrm{g} / \mathrm{ml}) ;$ C. tropicalis $(1.0 \mu \mathrm{g} / \mathrm{ml}) ;$ Saccharomyces cerevasiae $(2.0$ $\mu \mathrm{g} / \mathrm{ml})$ and Saccharomyces kluyverii $(3.0 \mu \mathrm{g} / \mathrm{ml})$. C. glabrata and C. norevegensis had dose dependent sensitivities of (MICs $12.0 \mu \mathrm{g} / \mathrm{ml}$ and $8.0 \mu \mathrm{g} / \mathrm{ml}$ ). C. albicans showed sensitivities of (MICs $=0.99 \mu \mathrm{g} / \mathrm{ml}$; $1.06 \mu \mathrm{g} / \mathrm{ml}$ ) in Rukungiri and Masaka Districts. In Mbarara District the most sensitive yeast strain to Amphotericin B were C. glabrata and $C$. sake $(\mathrm{MIC}=0.25 \mu \mathrm{g} / \mathrm{ml})$, followed by $C$. tropicalis and Saccharomyces kluyverii $(\mathrm{MIC}=0.38 \mu \mathrm{g} / \mathrm{ml})$. C. norvegensis and Saccharomyces cerevesiae were resistant to Amphotericin B (MICs $=0.75 \mu \mathrm{g} / \mathrm{ml} \& 9.0 \mu \mathrm{g} / \mathrm{ml})$.

District of isolation of yeast strains significantly $(p<0.05)$ impacted on MIC results as different MIC values were obtained after testing similar yeast strains from different districts against same anti-fungal agents. Thus, intra-species variations in average MIC values were noted among the yeast strains from different Districts. Itraconazoles tested against $C$. albicans showed varying MICs values of: $0.05 \mu \mathrm{g} / \mathrm{ml}$ in Mbarara District; $0.03 \mu \mathrm{g} / \mathrm{ml}$ in Rukungiri District and $0.14 \mu \mathrm{g} / \mathrm{ml}$ in Masaka District. Fluconazole tested against $C$. albicans also showed varying MICs of: 0.5 in Mbarara District; $0.99 \mu \mathrm{g} / \mathrm{ml}$ in Rukungiri District and 0.14 $\mu \mathrm{g} / \mathrm{ml}$ in Masaka District. Itraconazole MIC tested against $C$. norvegensis was $2.0 \mu \mathrm{g} / \mathrm{ml}$ in Mbarara and Rukungiri Districts but was $0.125 \mu \mathrm{g} / \mathrm{ml}$ in Masaka District. Fluconazole tested against $C$. tropicalis showed different MICs of: $1.0 \mu \mathrm{g} / \mathrm{ml}$ in Mbarara District and $0.38 \mu \mathrm{g} / \mathrm{ml}$ in Rukungiri and Masaka Districts.

\section{Discussion}

Although cutting edge modern technologies have advanced overall knowledge of microbial infections with consequent better outcome, what microorganisms will do when challenged with different surface active agents is still vague and incompletely understood. Disease diagnostic precision remain far below the minimum threshold needed to yield meaningful and reliable outcome especially in resource poor settings. Understanding the idiosyncrasy or characteristic uniqueness an organism when challenged with antimicrobial agents is a hug challenge to many antibiotic stewardship programs in resource limited settings. Perhaps the unpredictability of microbial response to surface active agent's emphasis the need to embrace the use of tests that are more sensitive and specific for more result oriented outcome.

Oropharyngeal lesions remain significant health challenge (26) that HIV infected population must overcome for a better life, because there are many questions that needs hard-to-get answers about possible disease comorbidities that could delay or prolong the healing of such oropharyngeal lesions. Confirmatory investigations are needed to determine if prolong or delayed healing of oropharyngeal lesions could be linked to: drug-resistance (1), non-adherence to prescription guidelines (2)), abuse of antibiotics with sub-lethal doses (3-4), re-infection (5), progression of HIV disease to AIDS (1-2) or 
resistant opportunistic infections (27). With the increasing notorious nature of yeast as predominant opportunistic pathogens in oropharyngeal lesions of HIV/AIDS patients, it becomes necessary to determine the characteristic vulnerability of yeasts to routine prophylactic antifungal agents to achieve better intervention.

In this study, Candida albicans were sensitive to fluconazole, Itraconazole and Amphotericin B respectively (Table 1; Fig 1, 4-5). Dose dependent sensitivity to various antifungal agents used were also shown by Candida glabrata ( $\mathrm{MIC}=12.0)$, Candida sake $(\mathrm{MIC}=0.5)$ and Candida norevegensis (MIC=8.0) respectively (Fig 2). This may indicate possible emergence of antifungal drug resistance. Again, it is not clear why yeast strains development of dose dependent sensitivity were different in different TASO centres because disease management policies are presumed to be the same in these centres. The observation that same yeast species from different centres under same prescription and TASO management policy yielded different MIC, shows unique idiosyncratic nature of yeasts response to treatment regimen and this makes it hard to extrapolate results across centres. Thus inter regional surveillance to monitor the emergence and re-emergence of yeast susceptibility in a locale antimicrobial stewardship program is urgently needed.

While $C$. albicans remain the leading aetiology of candidiasis (28-30), reports that infections caused by other non albicans yeast species are globally gaining more research attention with species varying between different geographic regions. This is problematic, because resistance to fluconazole may also mean resistance to other azoles and resistance are commonly controlled by point mutation and efflux pumps (31-32). The rates of azole resistance may differ between institutions due to prescribing patterns of clinicians for both the treatment of and prophylaxis against pathogenic candidiasis (33). In India, $C$. tropicalis is the predominant species, and rates of fluconazole resistance may vary significantly (34). The prevalence of $C$. parapsilosis was similar to $C$. albicans in some Chinese hospitals (35). Fluconazole susceptibility is also highly variable between institutions, with some reporting as high as $50 \%$ in intensive care units (35).

In Table 1 and Fig 1-5, all yeast isolates showed dose dependent sensitivity (DDS), susceptibility to both azoles and polyene antifungals, while $C$. glabrata, $C$. norvegensis and Zygosaccharomyces species exhibited reduced susceptibly (SDD) to fluconazole and were resistant to Itraconazoles in all studied districts. While the advantages of prophylaxis in HIV infected population cannot be denied, emergence of sub-population showing reduced sensitivity may be announcing the imperatives of a new prophylactic algorithms in immunocompromised HIV infected population living in resource limited countries like Uganda. Again, while prophylactic medication usually have clearly visible entry plan for infected patients, exit plan are often not stated, omitted or ignored by drug prescribers in Uganda. Patients on prophylactic drugs therefore continue to use the drugs even when the efficacy of the drugs decreases (36).

Non-albicans species, including $C$. glabrata have known innate resistance to antifungal drugs $(4,37-$ 38). The discrepancy between this report (Table 1) of reduced susceptibility and known innate resistance 
of $C$. glabrata may be explained by a possibility of misdiagnoses with other phenotypically similar yeast strains. Thus, the phenotypic similarity between $C$. glabrata, $C$. nivariensis and $C$. bracarensis, could be misdiagnosed as $C$. glabrata in the absence of good confirmatory typing methods (39). There may be emerging generation of Candida glabrata which may not be highly resistant to conventional antifungals as previously anticipated because sensitivity to Candida glabrata reports are beginning to emerge from different geographical settings (37-38, 40-42). Reports of $C$. glabrata resistant from some African countries include Cameroon (43), Ethiopia (44) and Tanzania (45), while reduced or dose dependent sensitivity to $C$. glabrata has been reported in South Africa (43) and Nigeria (42). The result of these study is similar to most reported studies reporting that C. globate resistance/dose dependent sensitivity to fluconazole but selectively sensitive to other antifungal agents such as nystatin, and amphotericin $B$, which are similar to the result of this study. Despite this window of hope for non albicans $\mathrm{C}$ glabrata susceptibity, resistant strains has increased in patients presenting with candidiasis in recent years (46) with increased mortality rates (39).

To understand the emergence of this dose dependent sensitivity in Candida albicans and non-Candida albicans species cohabiting the microecology of the oropharyngeal cavity of HIV patients, Melo et al. (47), found high-frequency transfer of plasmid DNA when pathogenic and food-borne yeasts were grown together despite the fact that many pathogenic yeast species are asexual and therefore not involved in intra- or inter species mating. This high-frequency transfer of plasmid DNA could define the observed emergence of dose dependent sensitivity by oropharyngeal Candida albicans, Candida glabrata, Candida sake and Candida norevegensis respectively (Tables 1 ).

Micro evolutionary high-frequency transfer of plasmid DNA (through cell lyses, or cytoduction) has been proved between C. glabrata and Saccharomyces cerevesiae (48). Although factors that govern the evolutionary revolution of micro-organisms are not yet completely understood, it is thought that environmental and nutritional factors are key to this evolutionary revolution of micro-organisms that can make them change from the original characteristics of their ancestral genealogy (the wild type), to something new (48-49). Microbial evolution tends to remove with time what will not assist its bid to survive in a particular environment and acquire new characters that will assist the organism to manipulate its environment (50) thus accounting for the suggested emergence of dose dependent sensitivity in the reported yeast strains (51). In addition, despite the "asexual" nature of C. glabrata, a potential cryptic sexual life cycle could promote rare interspecies mating with $\mathrm{C}$ albicans and other non albicans species of Candida (52).

From (Table 1, fig 1-6), C. norvegensis, C glabrata, Saccharomyces cerevisiae and Zygosaccharomyces species isolated from different districts showed a resistant MIC to Itraconazole, and Amphotericin B respectively. Immunosuppression, previous exposure to oral azoles, Clinical, cellular and molecular factors are known to contribute to development of antifungal drug resistance (53-54). Clinical factors leading to treatment failure of refractory disease may be associated with the patient's immune status (55), pharmacology of the drugs (13), or type of fungal infection (8). Cellular factors could be the result of 
replacement of a susceptible strain with a more resistant species or the alteration of an endogenous strain (by mutation or gene expression) to a resistant phenotype.

Molecular factors responsible for the resistance phenotype in the cell identified has been reported to include over expression of two types of efflux pumps, mutation of the target enzyme, and alteration of other enzymes in the same biosynthetic pathway as the target enzyme $(53,11)$. Generally, azoles and polyenes actions are mainly directed against ergosterol, the major sterol of the fungal plasma membrane, which is analogous to cholesterol in mammalian cells, (13-14). The dosing schedule may have an effect on the development of resistance (4), since patients treated with intermittent therapy were more likely to develop resistance than those treated continuously. Primary resistance and secondary resistance have been documented in HIV-infected patients (56). Although symptomatic candidiasis develops more commonly due to $C$. albicans, candidiasis are known to be caused also by non-albicans Candida sp. Fluconazole-resistant species, Candida norvegensis, have recently been reported as pathogens in patients receiving fluconazole (41).

The polyenes are known class of amphipathic antifungal drugs that target membranes containing ergosterol forming a channel through which cellular potassium ions, leak and thereby destroying the proton gradient within the membrane $(11,57)$. Amphotericin $B$ has antioxidant effect in vivo, protecting fungal cells against oxidative attack from the host $(11,58)$. Reports of Amphotericin B resistance are limited, but it appears prolonged pre-exposure to azole antifungal drugs and severely immunocompromised patients, especially patients with cancer, are at the highest risk perhaps explained by an alteration of cellular membrane components (59). Amphotericin B-resistant clinical isolates of C. tropicalis have also been found $(11,58)$. Minimum inhibitory concentration (MIC) of tested antifungal agents (Table 1$)$ was significantly $(p<0.05)$ dependent on strains of Candida species isolated from oropharyngeal lesions of studied population. Thus, types of oropharyngeal yeast strains present in the oral lesions significantly $(p<0.05)$ impacted on the MIC values as well as the efficacy of antifungal agents tested.

There is evidence to suggest that intermittent versus continuous dosing, the amount of drug administered, the length of treatment (27), the immune status of the patient and alterations in the membrane structure or in the sterol-to-phospholipid ratio in the membrane may be associated with resistance $(53,11,58)$. The success or failure of antifungal drug therapy is dependent not only on the drug, but also on the immune status of the host and a combination of azoles and cytokines may be an important therapeutic strategy for resistant fungal infections in immunocompromised individuals.

Susceptibility of $C$. norvegensis were significantly $(p<0.05)$ dependent on types of antifungal used. The depicted different levels of exposure to various antifungal agents tested may be a pointer to emergence of resistant clones of oropharyngeal yeast strains among the HIV infected patients studied. The frequency of diagnosed fungal infections is known to have risen due to increase in the number of immunosuppressed patients resulting from the AIDS epidemic and increase in the frequency of recalcitrant infections to standard antifungal therapy (13-14), Susceptibility of isolates to antifungals 
varied and MIC values were significantly $(\mathrm{p}<0.05)$ dependent on district of sample collection. Thus, despite being treated with same guidelines by clinicians in the same TASO centres, patient's uptake and compliance to treatment guidelines were different, thus depicting different levels of exposure to antifungals agents and probable prescription pattern. Fluconazole-resistant Candida norvegensis, have been reported as pathogens in patients receiving fluconazole $(41,24,60)$. It has been suggested that selection for resistant strains occurs with azole treatment but nosocomial transmission and transmission of resistant strains between partners can also occur (53). The polyene are amphipathic drugs which intercalate into membranes containing ergosterol, forming a channel through which cellular components, especially potassium ions, leak and thereby destroying the proton gradient within the membrane $(57,24$, 60). Amphotericin B resistance in Candida spp. can develop in patients previously exposed to azoles (59). Several strains of fluconazole- and amphotericin B-resistant $C$. albicans have been found in HIV-infected patients who have received prolonged courses of azole developed resistance with azoles (59).

Underlying medical conditions of the immunocompromised HIV infected population studied together with type of antifungal used, district of yeast isolation, and strains of yeast isolates significantly $(p<0.05)$ impacted on the MIC values obtained in this investigation (Table 1). To justify the role of underlying medical conditions in the healing of oral lesions in the immunocompromised HIV infected population (60), Nube et al (61) summarised specific risk factors of delayed wound healing to include but not limited to: infection, ischemia, ulcer size, depth, and duration as well as probing to bone (or osteomyelitis), location of ulcer, sensory loss, deformity (and high plantar pressure), advanced age, number of ulcers present and renal disease with ulcer depth (or size), infection, and ischemia listed as the most common risk factors. When there is high-quality treatment protocols and guidelines, $66 \%-77 \%$ of foot ulcers will heal and the remaining group are unlikely to heal, thus supporting the fact that quality of treatment and care impacts on wound healing rate (61). Non healing wounds are prevalent in resource poor settings due to poor treatment protocol while healing or delayed healing wound are more prevalence in adequate resources settings (62).

\section{Abbreviations}

$\mathrm{MIC}=$ Minimum inhibitory concentration

TASO $=$ The AIDS Support Organizations

HIV = Human Immunodeficiency Virus

AIDS = Acquired Immunodeficiency syndrome

$\mathrm{OC}=$ Oropharyngeal Candidiasis

CLSI = Clinical Laboratory Standard Institute 
EUCAST $=$ EUropean Committee for Antimicrobial Susceptibility Testing

RBD $=$ Randomized Block Design

CRD = Completely Randomized Design

FLSD = Fisher's least significant difference

SDD $=$ sensitive dose dependent

$\mathrm{AP}=$ Amphotericin $\mathrm{B}$

Flu = Fluconazole

IT = Itraconazole

\section{Declarations}

\section{Ethics approval and consent to participate}

This study was approved by: Kampala International University, Western Campus, The AIDS Support Organization, Uganda National Council of Science and Technology, Kampala and research participants' informed consent. All consent were written and there were no verbal consent. Those who could not read or write were allowed to thumb print to show consent after the research were interpreted in local language. All participants and stakeholders consented to participate in this study after informed consent as required by the law of Uganda.

\section{Consent for publication}

All figures included are original pictures taken by authors during the course of the study. It does not need any consent by anybody to publish. However, the single author of this manuscript gave full consent for this manuscript to be published including others were acknowledged in this work.

\section{Availability of data and materials}

All data generated or analyzed during this study are included in the body of the text while figures have been attached as separate document for your perusal

\section{Funding}


This study was supported in part by Kampala International University Western Campus and The AIDS Support Organization, Kampala Uganda. The funding bodies played no role in the study apart from the fund provided

\section{Authors contributions:}

This is not applicable as this is a single author manuscript. All other state holders who are not authors are listed in the acknowledgement section

\section{Competing interests}

The author declare no competing interests.

\section{Authors' contributions}

Not applicable since this is a single author paper. All others who assisted and who are not authors are mentioned in the acknowledgement section

\section{Acknowledgements}

Professor Victoria Pazos, and Barrister (Mrs) Oteji Ezera Agwu for moral support. Dr Francis Tirwomwe and Mr Nkangi Luwanga for technical assistance.

\section{References}

1. Kyeyune F, Nankya I, Metha S, Akao J, Ndashimye E, Tebit DM, et al. JCRC Drug Resistance Working Group. Treatment failure and drug resistance is more frequent in HIV-1 subtype D versus subtype Ainfected Ugandans over a 10-year study period. AIDS. 2013 Jul 31;27(12):1899-909.

2. Semvua SK, Orrell C, Mmbaga BT, Semvua HH, Bartlett JA, Boulle AA. Predictors of non-adherence to antiretroviral therapy among HIV infected patients in northern Tanzania. PLoS ONE 2017, 12(12): e0189460.

3. Westhoff S, van Leeuwe TM, Qachach O, Zhang Z, van Wezel GP, Rozen DE. The evolution of no-cost resistance at sub-MIC concentrations of streptomycin in Streptomyces coelicolor. ISME J. 2017 May;11(5):1168-1178.

4. Kohanski MA, DePristo MA, Collins JJ. Sublethal antibiotic treatment leads to multidrug resistance via radical-induced mutagenesis. Mol Cell. 2010 Feb 12;37(3):311-20.

5. Bajwa S, Kulshrestha A. Fungal infections in intensive care unit: challenges in diagnosis and management. Ann Med Health Sci Res. 2013 Apr-Jun;3(2):238-44. 
6. Agwu E, Ihongbe JC, Tirwomwe JF, Pazos V, Tirwomwe M, Casadesus L. Appraisal of Oral Lesions status of HIV/AIDS Patients in South Western Uganda. Brazilian Journal of Oral Sciences 2008; 7, (26): 1591-1595

7. Agwu E, Ihongbe JC, Pazos V, Tirwomwe JF. Zero prevalence of parasites associated with oral lesions of HIV infected and AIDS patients in South Western Uganda. African Health Sciences 2009; 9(4): $239-244$

8. Agwu E, Ihongbe JC, McManus BA, Moran GP, Coleman DC, Sullivan DJ. 2011 Distribution of yeast species associated with oro-pharyngeal candidiasis in HIV-infected patients in Southwest Uganda. Medical Mycology.2011; 1-5.

9. Agwu E, Ihongbe JC, Ezeonwumelu JOC, Moazzam ML. Baseline burden and antimicrobial susceptibility of pathogenic bacteria recovered from oral lesions of HIV/AIDS patients in SouthWestern Uganda. Oral Science International, 2015; 12 (2): 59-66.

10. Roemer T, Krysan DJ. Antifungal drug development: challenges, unmet clinical needs, and new approaches. Cold Spring Harb Perspect Med. ; 2014, 4(5):a019703.

11. Lakhani P, Patil A, Majumdar S. Challenges in the Polyene- and Azole-Based Pharmacotherapy of Ocular Fungal Infections. J Ocul Pharmacol Ther. 2019 Feb 1; 35(1): 6-22. Published online 2019 Jan 29.

12. Terrell CL, Hughes CE. Antifungal agents used for deep-seated mycotic infections. Mayo. Clin. Proc.1992; 67:69-91.

13. Xiong Z-Q, Tu X-R, Wei S-J, Huang L, Li X-H, Lu H, et al. (2013) The Mechanism of Antifungal Action of a New Polyene Macrolide Antibiotic Antifungalmycin 702 from Streptomyces padanusJAU4234 on the Rice Sheath Blight Pathogen Rhizoctonia solani. PLoS ONE 8(8): e73884.

14. Lv QZ, Yan L, Jiang YY. The synthesis, regulation, and functions of sterols in Candida albicans: Wellknown but still lots to learn. Virulence. 2016; 7(6):649-59.

15. Stefanini I, Rizzetto L, Rivero D, Carbonell S, Gut M, Heath S, et al. Deciphering the mechanism of action of 089, a compound impairing the fungal cell cycle. Scientific Reportsvolume8, Article number: 5964 (2018)

16. Lyman CA, Walsh TJ. Systemically administered antifungal agents. A review of their clinical pharmacology and therapeutic applications. Drugs 1992, 44:9-35.

17. Horter S, Thabede Z, Dlamini V, Bernays S, Stringer B, Mazibuko S, et al. "Life is so easy on ART, once you accept it": Acceptance, denial and linkage to HIV care in Shiselweni, Swaziland, Social Science \& Medicine, Vol. 176, March 2017, Pages 52-59

18. Tarquinio SBC, Oliveira LJC, Correa MB, Peres MA, Peres KG, Gigante DP, et al. Factors associated with prevalence of oral lesions and oral self-examination in young adults from a birth cohort in Southern Brazil. Cad. Saúde Publication. 2013.

19. Layer EH, Kennedy CE, Beckham SW, Mbwambo JK, Likindikoki S, Davis WW, et al. (2014) Multi-Level Factors Affecting Entry into and Engagement in the HIV Continuum of Care in Iringa, Tanzania. PLoS ONE 2014; 9(8): e104961. 
20. Wang S, Wang H, Ren B, Li X, Wang L, Zhou H, et al. Drug resistance of oral bacteria to new antibacterial dental monomer dimethylaminohexadecyl methacrylate. Sci Rep. 2018 Apr 3;8(1):5509.

21. EC, Clearinghouse on oral problems related to HIV infection and WHO Collaborating Centre on oral manifestations of the human immunodeficiency virus. (1993). Classification and diagnostic criteria for oral lesions in HIV infection. J. Oral Pathol. Med. 22: 289-291.

22. Sahand IH, Moragues MD, Eraso E, Villa-Vidal M, Quindos G, Ponton, J. Supplementation of CHROMagar Candida medium with Pal's medium for rapid identification of Candida dubliniensis. Journal of Clinical Microbiology. 2005, 43 (11): 5768-5770.

23. CLSI, Reference method for broth dilution antifungal susceptibility testing of filamentous fungi; Approved standard. (Second edition M38-A2)CLSI, Wayne, PA; 2008: 28

24. Arendrup M, Guinea, J, Cuenca-Estrella M, Meletiadis J, Mouton JW., Lagrou, K.Subcommittee on Antifungal Susceptibility Testing (AFST) of the ESCMID European Committee for Antimicrobial Susceptibility Testing methods document (EUCAST). EUCAST technical note on isavuconazole breakpoints for Aspergillus, itraconazole breakpoints for Candida and updates for the antifungal susceptibility testing method documents. Clin Microbiol Infect. 2016 Jun;22(6):571.e1-4.

25. Randomized Block Designs. 5.3.3.2. NIST/SEMATECH e-handbook of statistical methods; 2006. http://www.itl.nist.gov/div898/handbook [cited 2006].

26. Lacovou E, Vlastarakos PV, Papacharalampous G, Kampessis G, Nikolopoulos TP. Diagnosis and treatment of HIV-associated manifestations in otolaryngology. Infect Dis Rep. 2012 Jan 2;4(1):e9.

27. Bashir A, Garba I, Aliero AA, Kibiya A, Abubakar MH, Ntulume I et al. Superbugs-related prolonged admissions in three tertiary hospitals, Kano State, Nigeria PanAfrican Medical Journal 32, 166, 09/04/2019 April 9, 2019. 2019;32:166.

28. Wiederhold, NP, Antifungal resistance: current trends and future strategies to combat, Infection and Drug 2017, Vol 10, pp 249-259,

29. Pfaller MA, Diekema DJ, Gibbs DL, Newell VA, Ellis D, Tullio V. et al Results from the ARTEMIS DISK global antifungal surveillance study, 1997 to 2007: a 10.5-year analysis of susceptibilities of Candidaspecies to fluconazole and voriconazole as determined by CLSI standardized disk diffusion. J Clin Microbiol. 2010;48(4):1366-1377

30. Pfaller MA, Messer SA, Moet GJ, Jones RN, Castanheira M. Candidabloodstream infections: comparison of species distribution and resistance to echinocandin and azole antifungal agents in intensive care unit (ICU) and non-ICU settings in the SENTRY Antimicrobial Surveillance Program (2008-2009). Int J Antimicrob Agents. 2011;38(1):65-69

31. Enwuru CA, Ogunledun A, Idika N, Enwuru NV, Ogbonna F, Aniedobe M et al. Fluconazole resistant opportunistic oro-pharyngeal Candidaand non- Candida yeast-like isolates from HIV-infected patients attending ARV clinics in Lagos, Nigeria.Afr Health Sci. 2008;8(3):142-8.

32. Sanglard Emerging threats in antifungal-resistant fungal pathogens. Front Med (Lausanne). 2016;3:11 
33. Oberoi JK, Wattal C, Goel N, Raveendran R, Datta S, Prasad K. Non-albicans Candidaspecies in blood stream infections in a tertiary care hospital at New Delhi, India. Indian J Med Res. 2012;136(6):9971003

34. Chakrabarti A, Sood P, Rudramurthy SM, Chen S, Kaur H, Capoor M, et al. Incidence, characteristics and outcome of ICU-acquired candidemia in India. Intensive Care Med. 2015;41(2):285-295

35. Xiao M, Fan X, Chen SC, Wang H, Sun ZY, Liao K, et al. Antifungal susceptibilities of Candida glabrataspecies complex, Candida krusei,Candida parapsilosis species complex and Candida tropicalis causing invasive candidiasis in China: 3 year national surveillance. J Antimicrob Chemother. 2015;70(3):802-810

36. Brauner A, Fridman O, Gefen Nathalie O, Balaban Q. Distinguishing between resistance, tolerance and persistence to antibiotic treatment, Nature Reviews Microbiologyvolume14, pages320330 (2016) |

37. Sadeghi G, Ebrahimi-Rad M, Mousavi SF, Shams-Ghahfarokhi M, Razzaghi-Abyaneh M. Emergence of non-Candida albicans species: Epidemiology, phylogeny and fluconazole susceptibility profile. J Mycol Med.2018 Mar;28(1):51-58.

38. Miranda-Cadena K, Marcos-Arias C, Mateo E, Aguirre JM, Quindós G, Eraso E. Prevalence and antifungal susceptibility profiles of Candida glabrata, Candida parapsilosis and their close-related species in oral candidiasis. Arch Oral Biol.2018 Nov;95:100-107.

39. Yoo Jl, Choi CW, Lee KM, Lee YS. Gene Expression and Identification Related to Fluconazole Resistance of Candida glabrataOsong Public Health Res Perspect. 2010;1(1):36-41. 10.1016/j.phrp.2010.12.009

40. Figueiredo-Carvalho MHG, Ramos LS, Barbedo LS, de Oliveira JCA, Dos Santos ALS, Almeida-Paes R, et al. Relationship between the Antifungal Susceptibility Profile and the Production of VirulenceRelated Hydrolytic Enzymes in Brazilian Clinical Strains of Candida glabrata. Mediators Inflamm. 2017;2017:8952878.

41. Sandven P, Nilsen K, Digranes A, Tjade T, Lassen, J. Candida norvegenesis: a fluconazole-resistant species. Agents. Chemother. 1997; 41:1375-1376.

42. Inyang NJ, Enweani IB, Agwu E, Esumeh FI, Obiazi HAK, Eifediyi RA, et al. Colonization and susceptibility profile of vaginal yeast isolates in Ekpoma, Edo state Nigeria. Special Fungal Pathogens Journal (SBPJ) 2015; Vol 1, No 1: p 0007-0013.

43. Dos Santos Abrantes PM, McArthur CP, Africa CWJ. Multi-drug resistant oral Candidaspecies isolated from HIV-positive patients in South Africa and Cameroon. Diagn Microbiol Infect Dis.2014; 79(2):222-7.

44. Mulu A, Kassu A, Anagaw B, Moges B, Gelaw A, Alemayehu M,et al. .Frequent detection of ' azole' resistant Candida species among late presenting AIDS patients in northwest Ethiopia. BMC Infect Dis. 2013;13:82. 10.1186/1471-2334-13-82

45. Hamza OJ, Matee MI, Moshi MJ, Simon EN, Mugusi F, Mikx FH, et al. Species distribution and in vitro antifungal susceptibility of oral yeast isolates from Tanzanian HIV-infected patients with 
primary and recurrent oropharyngeal candidiasis. BMC Microbiol. 2008;8:135. 10.1186/1471-2180-8135

46. Vermitsky JP, Edlind TD: Azole resistance in Candida glabrata: coordinate upregulation of multidrug transporters and evidence for a Pdr1-like transcription factor.Antimicrob Agents Chemother.2004;48(10):3773-81. 10.1128/AAC.48.10.3773-3781.2004

47. Melo NR, Taguchi H, Jorge J, Pedro RJ, Almeida OP, Fukushima K. et al. Transfer of Genetic Material between Pathogenic and Food-Borne Yeasts. Appl. Environ. 2006; 72(7): 5122-5125.

48. Davenport ER, Sanders JG, Song SJ, Amato KR, Clark AG, Knight R. The human microbiome in evolution. BMC Biol. 2017 Dec 27;15 (1):127.

49. Dujon B, Sherman D, Fischer G, Durrens P, Casaregola S, Lafontaine I. Genome evolution in yeasts. Nature 2004; 430:35-44.

50. Foster KR, Schluter J, Coyte KZ, Rakoff-Nahoum S. The evolution of the host microbiome as an ecosystem on a leash. Nature. 2017 Aug 2;548(7665):43-51.

51. Borenstein, E, Shlomi T, Ruppin E, Sharan R. Gene loss rate: a probabilistic measure for the conservation of eukaryotic genes. Nucleic Acids Research 2007; 35(1): e7.

52. Wong S, Fares MA, Zimmermann W, Butler G, Wolfe KH. Evidence from comparative genomics for a complete sexual cycle in the 'asexual' pathogenic yeast Candida glabrata. Biol. 2003; 4:R10-R19.

53. White TC, Marr KA, Bowden RA. Clinical, cellular, and molecular factors that contribute to antifungal drug resistance. Microbiol. Rev. 1998; 11:382-402.

54. Vazquez L. Antifungal Prophylaxis in Immunocompromised Patients. Mediterr J Hematol Infect Dis. 2016 Sep 1;8(1):e2016040.

55. Sulaiman W, Abdullah AC, Tan-Tsen-Chuen J, Baba S, Karim N. Refractory Adult Onset Still's Disease. Cureus. 2017 Oct 25;9(10):e1802.

56. Moran GP, Sullivan DJ, Henman MC, McCreary CE, Harrington BJ, Shanley DB, Coleman DC. Antifungal drug susceptibilities of oral Candida dubliniensis, isolates from human immunodeficiency virus (HIV)-infected and non HIV-infected subjects and generation of stable fluconazole-resistant derivatives in vitro. Agents Chemother. 1997; 41:617-625.

57. Vanden-Bossche H, Marichal P, Odds FC. Molecular mechanisms of drug resistance in fungi. Trends Microbiol. 1994; 2:393-400

58. Radwan MA, AlQuadeib BT, Šiller L, Wright MC, Horrocks B. Oral administration of amphotericin B nanoparticles: antifungal activity, bioavailability and toxicity in rats,Drug Delivery, 2017; 24:1, 40-50,

59. Kelly SL, Lamb DC, Kelly DE, Loeffler J, Einsele H. Resistance to fluconazole and amphotericin in Candida albicans from AIDS patients. Lancet 1996; 348:1523-1524

60. Shokohi T, Badali H, Amirrajab N, Ataollahi MR, Kouhpayeh SA, Afsarian MH. In vitroactivity of five antifungal agents against Candida albicans isolates, Sari, Iran. Curr Med Mycol. 2016 Jun; 2(2):3439. 
61. Nube V, Frank G, White J, Stubbs S, Nannery S, Pfrunder L, et al. Hard-to-heal diabetes-related foot ulcers: current challenges and future prospects. Chronic Wound Care Management and Research » 2016; (3): 133-146

62. Agwu E, Ihongbe JC, Inyang NJ. Prevalence of Quinolone susceptible Pseudomonas aeruginosa and Staphylococcus aureus in delayed-healing diabetic foot ulcers in Ekpoma Nigeria. Wounds 2010; 4: 100-105.

\section{Tables}

Table 1: Break-point mean Minimal Inhibitory Concentration (MIC) of Azoles and Polyene against selected representative Candida strains from the population surveyed $n=144$

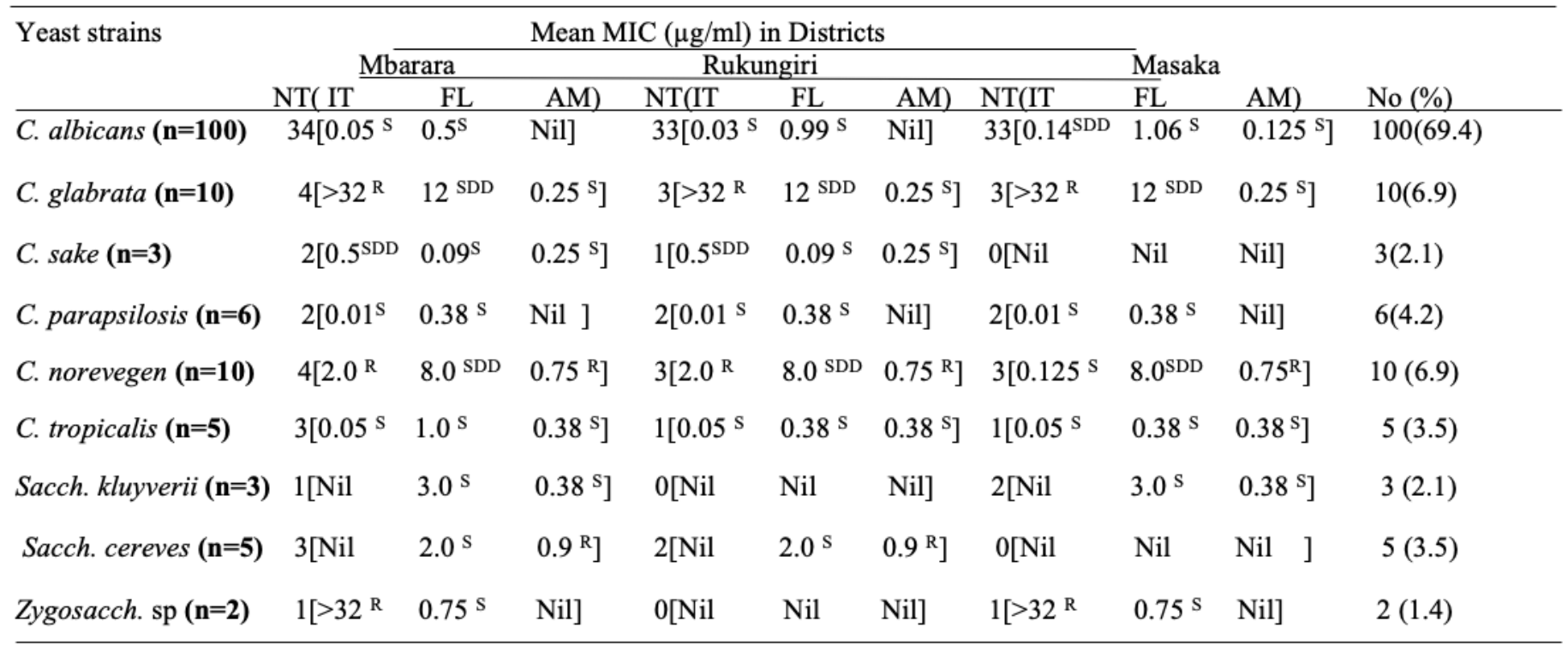

IT = Itraconazole, $\mathbf{N T}=$ No of isolates tested, $\mathbf{F L}=$ Fluconazole, $\mathbf{A M}=$ Amphotericin B, Sacch. = Saccharomyces, $\mathbf{S}=$ Sensitive, $\mathbf{S D D}=\mathbf{S e n s i t i v i t y ~ i s ~ D o s e ~}$ Dependent, $\mathbf{R}=$ Resistant, $\mathbf{N i l}=$ Antibiotics not tested against the corresponding fungi. Using Randomized Block Design ( $\alpha=0.05$ ), no significant difference $(p>0.05)$ in MIC observed between district (a); there was a significant difference $(p<0.05)$ in MIC between yeast strains (b) and no significant difference $(p>0.05)$ in the interaction of different antifungal agents used against different yeasts in the districts surveyed $(c)$. $n=$ total number tested. $a=0.00, b=3.905, c=0.001$. MIC 90 is the MIC at which $90 \%$ of tested isolates are inhibited by agents

\section{Conclusions}

In conclusion Candida albicans remain significantly susceptible to tested antifungal agents. Emerging dose dependent susceptibility in non albicans oropharyngeal yeast strains is interesting in this era of increasing treatment failures due to drug resistance. Pattern of resistance recorded may suggest significant contribution of yeast in the recurrent or delayed oropharyngeal lesions of HIV population studied. Sustained surveillance with MIC which impacts treatment outcome is recommended.

\section{Figures}




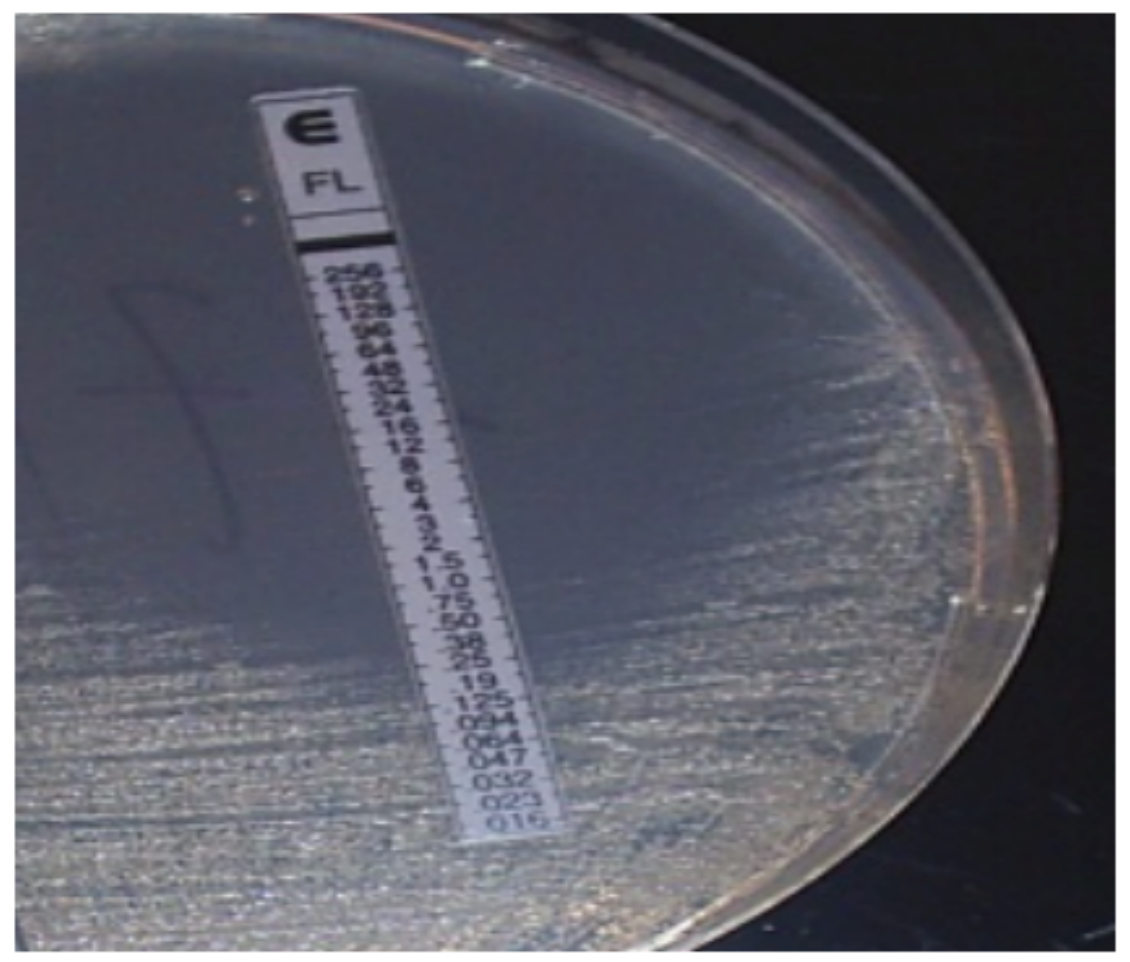

Figure 1

Fluconazole= sensitive 0.5

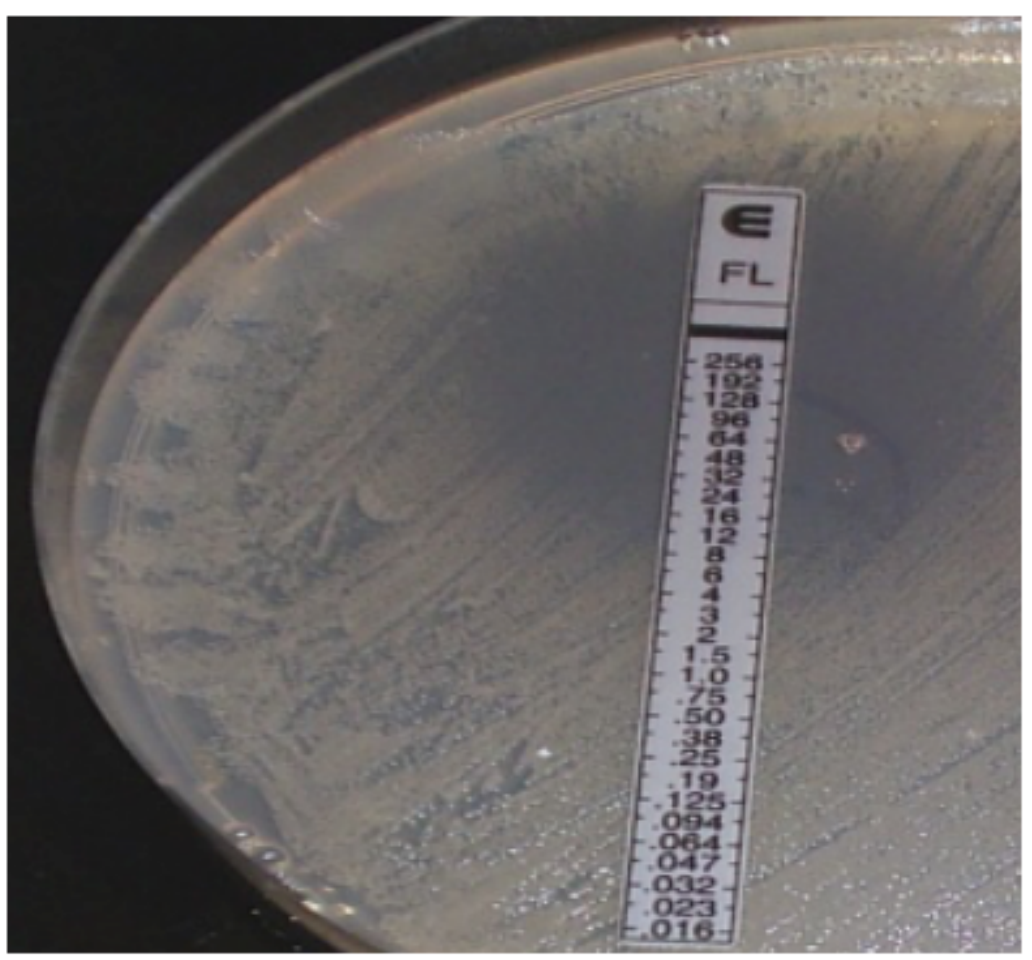

Figure 2

Fluconazole $=12$ SDD 


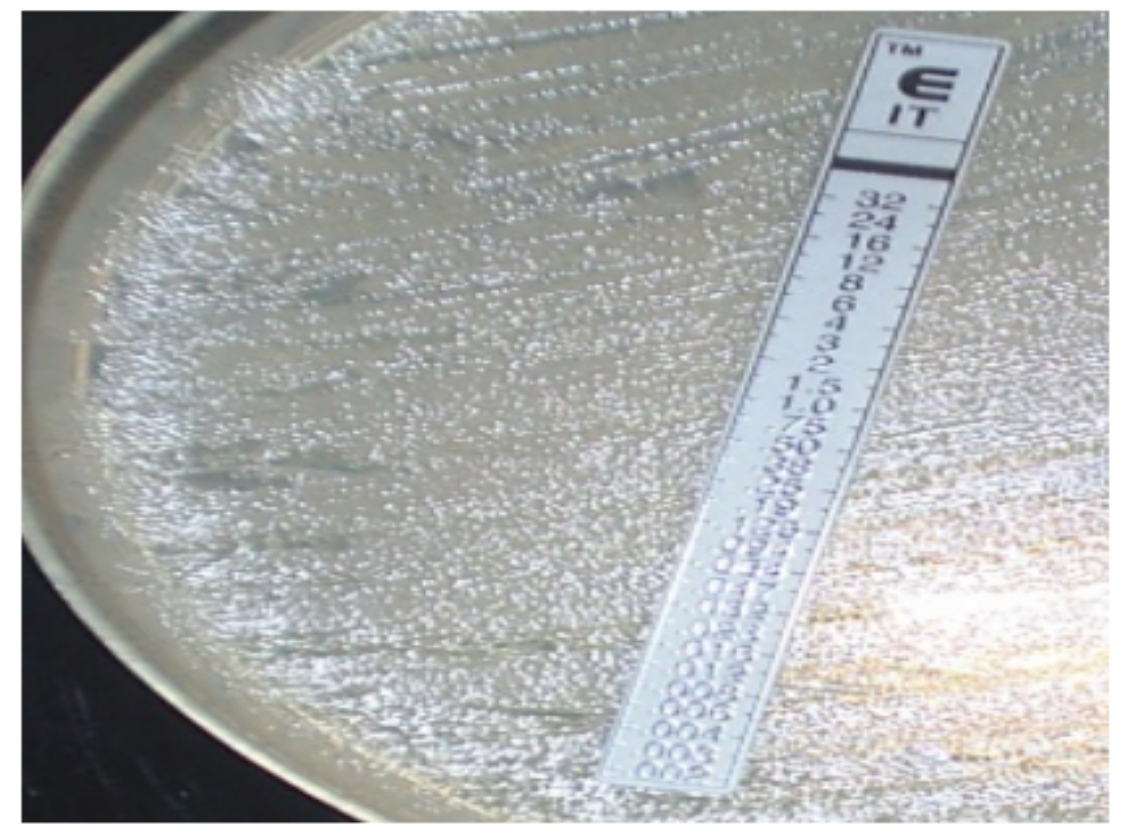

Figure 3

Itraconazole MIC = 0 is resistance

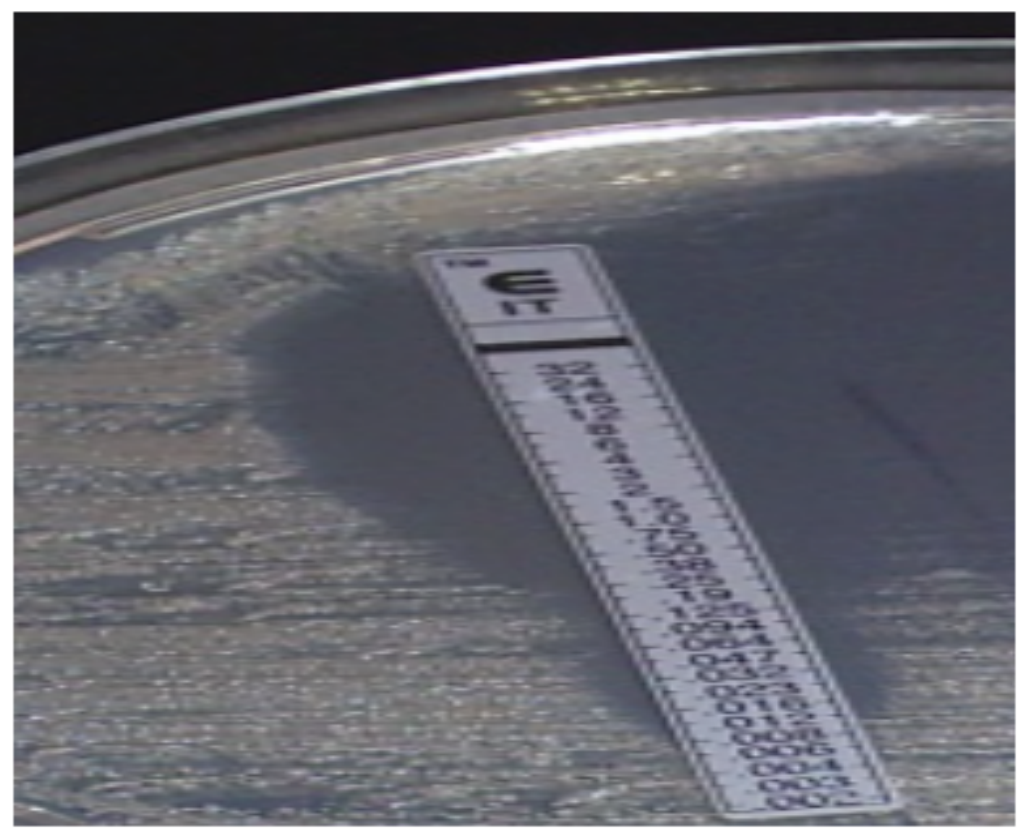

Figure 4

Itraconazole MIC $=0.064$ sense 


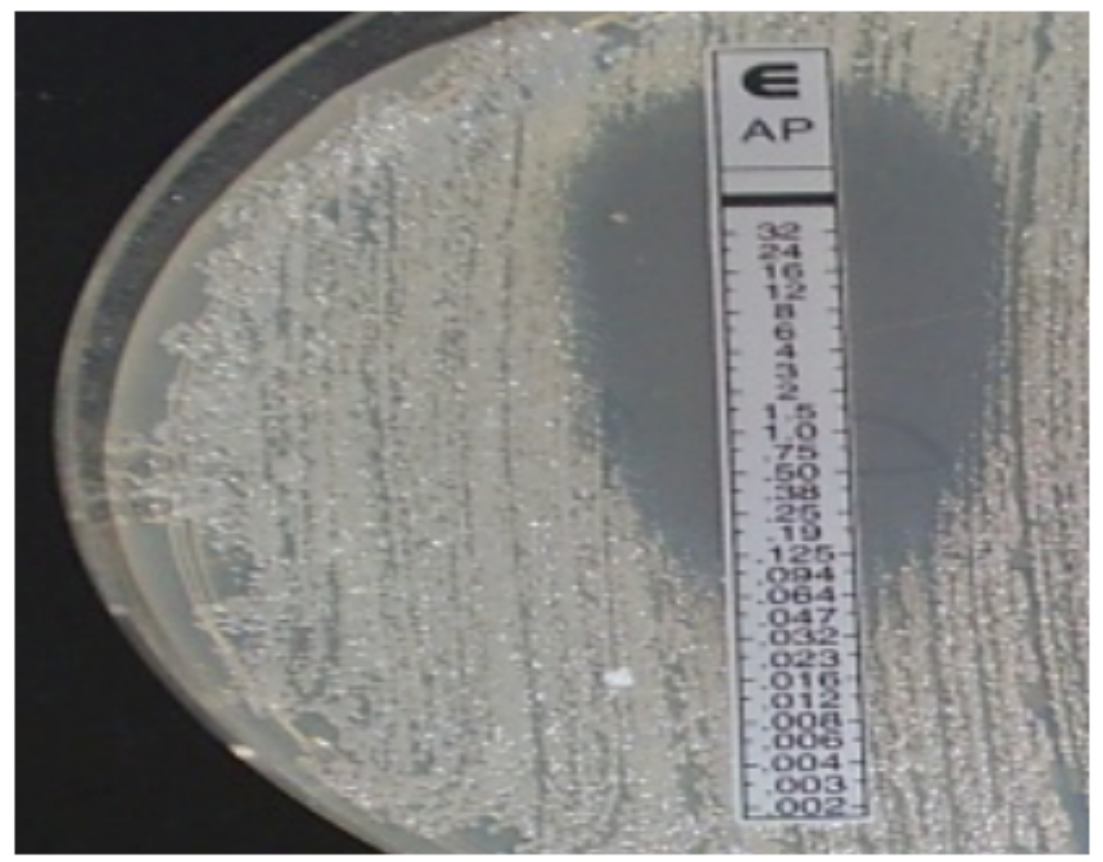

Figure 5

AP MIC $=0.1$ Sens

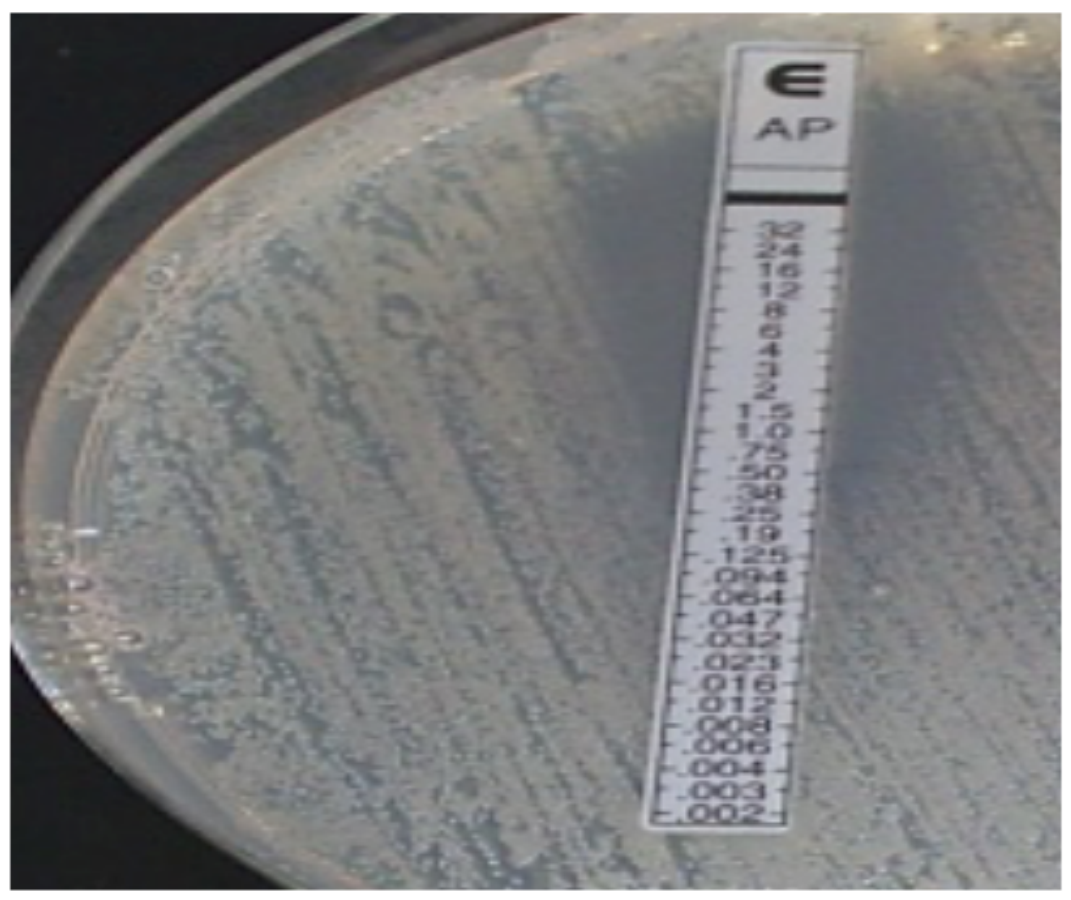

Figure 6

AP: MIC = 2 Resistant 